\title{
Plurilinguisme, multimodalité et compétence d'interaction: parler de nombres dans des interactions commerciales entre locuteurs de langues romanes
}

\author{
Vanessa Piccoli \\ CNRS - LabEx ASLAN \\ Laboratoire ICAR UMR 5191 ENS de Lyon \\ vanessa.piccoli@ens-lyon.fr
}

\begin{abstract}
Résumé. En adoptant une approche conversationnaliste, cette contribution étudie comment les participants parlent de prix et d'autres quantités numériques exactes lors de rencontres commerciales entre des locuteurs de langues romanes. Par le biais d'une analyse séquentielle et multimodale de données audiovisuelles d'interactions naturelles, cet article montre que, quand ils parlent de nombres, les participants utilisent une variété spécialement large de pratiques pour atteindre l'intercompréhension : des gestes, à la mobilisation de supports écrits, au recours à d'autres langues. Faisant ainsi, ils montrent leur créativité, leur flexibilité et leur conscience par rapport au contexte de communication. Nous considérons ainsi ces pratiques plurilingues et multimodales comme des marques d'une compétence d'interaction plurilingue.
\end{abstract}

\begin{abstract}
Plurilingualism, multimodality and interactional competence: talking about numbers in commercial interactions between Romance speakers. Grounding on a conversation analytic approach, this contribution explores how participants talk about prices and other exact numbers in commercial encounters between Romance speakers. Through a sequential and multimodal analysis of audio-visual data of naturally occurring interactions, this paper shows that, when speaking about numbers, participants use an especially large variety of practices to ensure intercomprehension: from gestures, to the mobilization of written materials, to the use of other languages. By doing so, they show creativity, flexibility and mindfulness about the particular context of communication. We argue that these plurilingual and multimodal practices can be seen as signs of a plurilingual interactional competence.
\end{abstract}




\section{Introduction}

Dans les salons commerciaux les participants parlent souvent de nombres : ils mentionnent des prix, des quantités de produits, des mesures, des dates. La bonne compréhension de ces nombres constitue un enjeu important dans une interaction entre des professionnels qui cherchent à instaurer une nouvelle relation commerciale. Un malentendu sur le prix ou sur le nombre de produits commandé peut avoir des répercussions négatives importantes sur cette relation et mettre en péril une collaboration future. Par conséquent, les participants ont intérêt à être spécialement attentifs à la compréhension mutuelle quand ils parlent de nombres, surtout s'ils se trouvent dans une situation de communication plurilingue, comme celle d'un salon international.

Dans cette contribution, nous analysons des séquences de conversation naturelle où les participants discutent de nombres au sein de trois salons commerciaux internationaux, en France et en Italie. Notre but est de participer à une description approfondie du fonctionnement de la communication en contextes de plurilinguisme et, plus particulièrement, entre des locuteurs de langues romanes. Nous considérons en effet ces séquences comme des lieux d'observation privilégiés des pratiques multimodales et variées que les participants utilisent pour atteindre une communication efficace. De plus, nous considérons que l'analyse de ces pratiques peut servir à développer une réflexion didactique sur la compétence d'interaction en langues secondes.

\section{Cadre théorique et méthodologique}

Cette étude a été réalisée dans le cadre d'une recherche doctorale [1] visant à étudier la communication plurilingue entre locuteurs de langues romanes différentes, par le biais d'une approche interactionniste, dans le but de contribuer à l'avancement des études sur l'intercompréhension romane. En effet, bien que ces études - qui se sont développées dans le domaine de la didactique des langues à partir des années 80 (pour une revue voir $[2,3]$ ) reposent sur l'idée qu'entre locuteurs de langues romanes il est possible de communiquer en parlant chacun sa propre langue, aucune recherche n'avait jusque-là étudié le fonctionnement de l'intercompréhension orale dans une situation naturelle. Notre recherche a donc abordé cet aspect, à travers une analyse d'interactions spontanées entre locuteurs romanophones dans une situation non-didactique, notamment celle des salons commerciaux internationaux.

Notre étude se situe ainsi dans le domaine de l'analyse conversationnelle [4] et plus précisément dans la lignée des études dédiées à la communication dans des situations de travail plurilingue $([5,6,7]$ entre autres). Ces recherches ont montré que dans des situations institutionnelles les participants sont généralement orientés vers la réalisation d'une tâche communicative et que, par conséquent, ils tendent à se concentrer sur le but, plutôt que sur la forme de la communication. En adoptant la terminologie de Drew et Heritage [8], on peut définir ces conversations comme étant goal-oriented, orientées vers un but. Comme Firth [9] l'a montré pour les interactions en anglais lingua franca, quand les participants sont orientés vers l'efficacité communicative non seulement ils laissent passer les productions maladroites de leur interlocuteur, mais ils travaillent même à normaliser le discours de l'autre. Si cette attitude peut mener à une «linguistically lawless lingua franca jungle » ([10]: 159), elle est pourtant efficace du point de vue communicatif. Ainsi, dans ces interactions, généralement les rôles de locuteur natif et non-natif ne semblent pas être traités comme pertinents par les interactants, qui mettent en avant plutôt leurs identités professionnelles. Les participants peuvent en effet mobiliser plusieurs identités au cours d'une même interaction. Kurhila [11] a par exemple montré que dans des interactions entre clients (non-natifs) et secrétaires (natifs) dans une école de langue, les catégories professionnelles sont généralement traitées comme plus pertinentes par rapport aux identités linguistiques et que ces dernières ne sont mobilisées que par les non-natifs. Les 
clients ont la possibilité de mobiliser leurs différentes identités selon leurs besoins, en s'orientant dans certains cas vers leur non-compétence (par exemple, en montrant des restrictions dans leur capacité de production verbale). Cependant, cela ne met pas en discussion leur compétence institutionnelle par rapport à la tâche à accomplir : « [e]ven if portraying herself as linguistically incompetent, the SL-speaker can display institutional competence » $([11]: 71)$.

Dans le cadre des interactions institutionnelles entre locuteurs de plusieurs langues, l'efficacité communicative semble donc être l'enjeu principal. Pour aboutir à cette efficacité, les locuteurs peuvent recourir à un grand nombre de pratiques et, quand plusieurs langues sont disponibles, ils peuvent les alterner et les mélanger selon leurs besoins. Les nombreux travaux qui ont été développés au sein du projet DYLAN $([12,13]$ entre autres) ont mis en évidence le niveau très fin de coordination que les participants utilisent pour gérer la participation dans des groupes de travail plurilingue. Le travail interactionnel des participants vise à permettre la progression de l'activité en cours (principle of progressivity) et, en même temps, à garantir la compréhension mutuelle entre les participants (principle of intersubjectivity). Pour atteindre ces deux buts, les locuteurs peuvent avoir recours à une vaste gamme de ressources multimodales. L'organisation de l'interaction se fonde alors sur " the use of the available linguistic resources and on a locally situated linguistic creativity » ([7]: 10). Autrement dit, les participants s'orientent vers l'« exploitation des multiples ressources linguistiques à leur disposition » ([6]: 3).

Notre étude partage avec les études du projet DYLAN l'observation du caractère dynamique, ingénieux et localement situé de la communication plurilingue. Par ailleurs, la proximité des langues romanes entraîne des spécificités : les participants peuvent arriver à comprendre la langue de l'autre lors d'une émergence ponctuelle - par exemple, lorsqu'un locuteur recourt à sa L1 durant une recherche de mot [14] - ou même, dans certains cas, lors de longues séquences de communication plurilingue explicitement négociées [15]. Dans cette contribution, nous nous concentrerons sur un cas d'émergence ponctuelle : le recours à la L1 des locuteurs, en combinaison avec des pratiques multimodales, à l'intérieur d'un micro-environnement qui semble favoriser le plurilinguisme, c'est-à-dire le discours sur les quantités numériques.

\subsection{Une perspective didactique}

Si les études interactionnistes sur les situations professionnelles plurilingues ont avant tout une fonction descriptive, elles ne sont pas dépourvues d'implications pour la didactique des langues. L'attention portée à la multimodalité et aux phénomènes d'alternance et de mélange de langues contribue en effet à surmonter le «biais linguistique »[16] et à remettre en discussion la vision monolingue de l'enseignement des langues. En particulier, l'approche conversationnaliste a participé à la redéfinition de la notion de compétence linguistique.

Au cours des dernières décennies, on a assisté à une prise de conscience progressive dans le domaine de l'enseignement/apprentissage des langues secondes : les compétences d'un locuteur en langues secondes ne peuvent pas être réduites à la dichotomie entre compétences de production et de réception. La nature même de l'interaction rend nécessaire la prise en compte d'une autre compétence, qui consiste notamment à savoir interagir avec les autres, et qui a été désignée comme compétence d'interaction. Dans les trois dernières décennies, un nombre de plus en plus important de travaux a été dédié à l'étude de la compétence d'interaction ([17, 18, 19, 20, 21] entre autres). Mais bien que les travaux théoriques soient de plus en plus nombreux, leur application dans la pratique de l'enseignement et de l'évaluation des langues secondes semble encore loin d'être accomplie [22]. La compétence d'interaction, reposant sur les contingences interactionnelles et sur l'entente avec l'interlocuteur, est difficilement mesurable et sa prise en compte dans les programmes et les tests linguistiques constitue un défi encore ouvert aujourd'hui. De plus, 
cette difficulté d'application semble être liée à l'absence d'une définition théorique claire et univoque de la notion. Comme le souligne Hauser [23], différentes façons de conceptualiser la compétence interactionnelle coexistent aujourd'hui. En particulier, le chercheur met en évidence deux oppositions qu'on retrouverait dans les travaux récents dans le domaine : en premier lieu, une vision de la compétence interactionnelle comme un objectif à atteindre à travers la participation à l'interaction s'opposerait à l'idée de celle-ci comme une condition nécessaire pour participer à l'interaction. Une deuxième opposition se retrouverait entre « interactional competence as something which is acquired and possessed by the individual and interactional competence as something which is co-constructed by all participants within the interaction $»([23]: 1)$.

S'il est vrai que ces différentes conceptualisations rendent difficile de cerner et de définir la compétence interactionnelle, elles ne sont pourtant pas forcément en contradiction les unes avec les autres. Au contraire, elles peuvent être vues comme les faces d'une même médaille. Ce qui émerge dans l'ensemble des études sur le sujet est que la compétence d'interaction serait à la fois nécessaire pour interagir avec les autres et atteinte grâce à l'interaction, à la fois une compétence individuelle et basée sur la collaboration avec les autres. Les différentes définitions de la compétence interactionnelle évoquent toujours cette double dimension - cognitive et interactionnelle, individuelle et collective -, même si elles peuvent mettre en lumière un aspect plutôt que d'autres. Ainsi pour Markee, la compétence d'interaction consiste à utiliser les ressources sémiotiques d'une langue pour « co-construct with their interlocutors locally enacted, progressively more accurate, fluent, and complex interactional repertoires in the L2» ([18] : 406). Pour Young, elle consiste dans " the construction of a shared mental context through the collaboration of all interactional partners » ([20]: 428). Pour Walsh, elle serait l'habileté « to interact with others and to collectively reach understandings » ([21]:2).

Mais si la notion de compétence d'interaction est normalement utilisée pour parler de la capacité d'un locuteur à s'exprimer dans une certaine L2, comment peut-on considérer cette compétence dans le cadre d'une interaction où les participants utilisent plusieurs langues à la fois ? Si l'on considère que la compétence d'interaction est « what is needed in order to 'survive' most communicative encounters » ([21]:2), on peut imaginer que pour 'survivre' quand plusieurs langues sont en jeu les locuteurs auront besoin de quelques stratégies spécifiques. Dans la description de ces spécificités, nous considérons que la contribution d'une étude basée sur des données authentiques - qui permet donc de tracer l'émergence de cette compétence dans les interactions - est spécialement pertinente, car elle fournit une base solide pour des applications didactiques. Dans cette contribution, nous chercherons ainsi à montrer l'émergence d'une compétence d'interaction plurilingue dans des séquences naturelles de discussion sur les nombres entre locuteurs de langues romanes différentes.

\section{Les données}

Notre analyse s'appuie sur un corpus audiovisuel réalisé en 2014 dans trois salons commerciaux internationaux (un salon de lingerie, un salon de vins et un salon de livres pour enfants), en France et en Italie, auprès d'exposants de trois langues maternelles différentes (italien, français, espagnol). Le corpus a été collecté dans le but d'étudier la communication spontanée entre locuteurs de langues romanes dans une situation nondidactique et notamment pour observer à quelles pratiques verbales et non verbales les locuteurs ont recours pour atteindre une communication efficace.

Les salons internationaux constituent une situation de fort plurilinguisme, où la langue de communication n'est pas préétablie, mais négociée par les participants dans chaque interaction. Recourir à l'anglais lingua franca - souvent considéré comme la langue de communication par défaut dans les relations de travail au niveau international - est une pratique courante, mais elle n'est pas la seule : dans notre corpus, l'anglais est adopté dans 
environ la moitié des interactions (voir [1]). Dans les interactions restantes, les participants parlent dans la langue de l'un des participants ou ils recourent à plusieurs langues à la fois. De plus, leur communication repose souvent fortement sur des ressources autres que le langage verbal.

Dans l'analyse, nous ne nous limiterons pas à analyser les productions verbales des participants, mais nous prendrons aussi en compte la composante multimodale de l'interaction, c'est-à-dire le système complexe de gestes, regards, mobilisation d'objets et déplacements dans l'espace que les locuteurs mettent en place aux cours de leurs interactions [24]. En considérant que le langage est indissociable des autres ressources et que, comme le dit Mondada, " [m]ultimodal resources are integrated in a holistic way and make sense together » ([25]: 139), nous n'attribuerons pas une hiérarchie a priori à ces éléments, mais nous regarderons plutôt la pertinence de ces ressources dans l'interaction. En adoptant une approche emique, nous analyserons notamment tous les phénomènes qui semblent être pertinents pour les participants à l'interaction.

Les données ont été transcrites selon la convention ICOR, développée au laboratoire ICAR, dans la lignée des indications de Jefferson [26]. Des notations supplémentaires ont été ajoutées pour rendre compte des pratiques multimodales des participants (voir Conventions de transcription). En revanche, pour les termes en italien, nous avons choisi de ne pas proposer une traduction insérée dans les transcriptions, mais de fournir plutôt des reformulations et des explications dans le corps de l'analyse, là où il nous a semblé nécessaire. Ce choix a été motivé d'une part par la volonté de préserver la lisibilité de la transcription, d'autre part par la considération que proposer une traduction serait quelque part en contradiction avec la notion d'intercompréhension romane que notre recherche veut promouvoir.

\section{Analyse}

Dans les salons commerciaux, les participants disent souvent des nombres. Qu'ils discutent de prix, de quantités ou des années de production de leurs produits, ils doivent fréquemment utiliser des numéraux. Les séquences de discussion sur les nombres constituent des moments assez spéciaux à l'intérieur du corpus, car elles ont des contraintes particulières. Si généralement les participants aux interactions ne prêtent pas beaucoup d'attention à l'exactitude de leurs productions, lorsqu'ils parlent de nombres leur attitude change. Quand ils indiquent une quantité numérique exacte, ils doivent être attentifs à la bonne formulation du nombre, pour être sûrs d'éviter des malentendus qui pourraient mettre en danger la réussite de la transaction commerciale et, peut-être, la construction d'une relation professionnelle de longue durée. En effet, puisque l'accès aux salons est réservé aux professionnels du secteur, l'enjeu principal de ces interactions ne consiste pas dans l'achat/vente d'un certain produit mais plutôt dans la prise de contacts pour des relations professionnelles futures (par exemple, entre un producteur et un distributeur ou entre un illustrateur et un éditeur). Il ne s'agit donc pas, par exemple, d'acheter une bouteille de vin, mais d'en acheter plutôt dix mille par an.

Prenons l'extrait suivant. Le producteur italien Carmine (CAR), sa fille Angelica (ANG) et Rosa (ROS), une copine d'Angelica qui a été embauchée pour les aider pendant le salon, ont un stand dans un salon de lingerie en France. Ils sont en train de parler avec trois clientes ${ }^{1}$ françaises (productrices de lingerie elles-mêmes) - Monique (MON), Béatrice (BEA) et Marine (MAR). Leur interaction s'est déroulée jusque là principalement en anglais, même si les participants recourent de temps en temps à des mots ou des

\footnotetext{
${ }^{1}$ Nous utilisons le terme générique de « client » pour indiquer toute personne qui se rend dans un stand pour se renseigner sur les produits et les tester, bien que l'achat direct des produits ne soit pas forcément le but de sa visite.
} 
expressions dans leurs langues maternelles - sans doute à cause d'une compétence plutôt faible en anglais. Juste avant le début de cet extrait, les clientes ont montré leur intérêt pour un certain type de soutien-gorge. Carmine, après avoir consulté un document en papier, leur annonce le prix du produit.

\section{Ex.1: Thirty-nine}

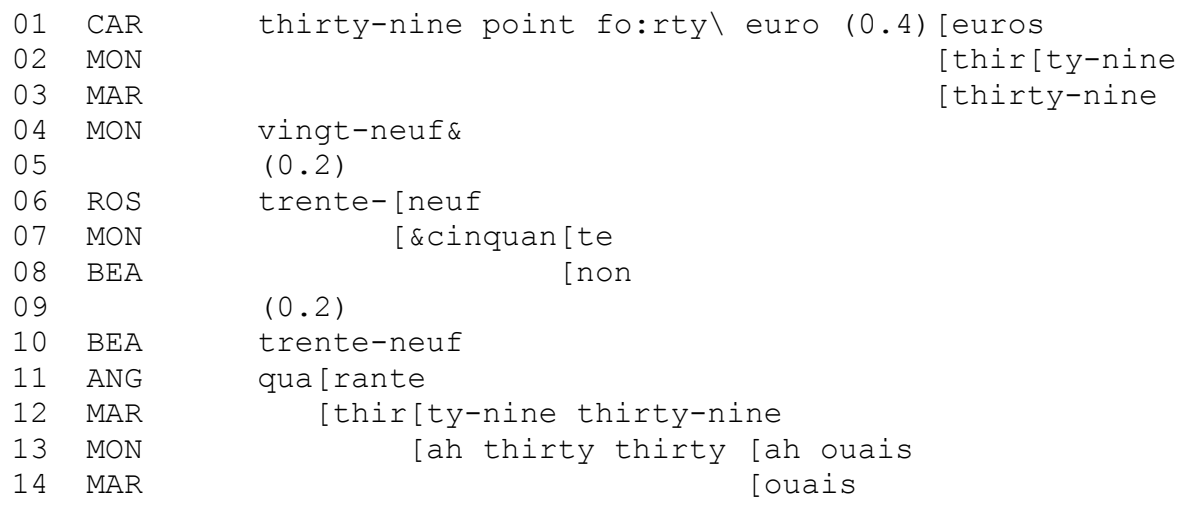

La bonne réception de l'information donnée par Carmine, c'est-à-dire le prix du soutiengorge, est très importante pour les participants, car de celle-ci pourrait dépendre une transaction commerciale de grande envergure. C'est pour cette raison que, quand Carmine annonce le prix (ligne 01, dorénavant 01), celui-ci est d'abord répété par deux des clientes (02-03) et puis traduit en français par Monique (04). Cependant, dans cette opération de traduction, Monique commet une erreur : elle traduit en effet "vingt-neuf» au lieu de «trente-neuf ». Cette erreur est relevée par les autres et plusieurs participants s'engagent dans une hétéro-réparation [27] : Rosa dit le prix correct en français (06); Béatrice produit une négation ( «non »-08) pour signaler à Monique son erreur et ensuite répète à son tour le nombre en français (10). Enfin Marine répète encore deux fois le nombre en anglais (12). De plus, Monica commet une deuxième erreur dans la formulation des centimes : elle dit « cinquante» (07) au lieu de « quarante» (le prix complet étant 39.40 euros). Cette deuxième imprécision est corrigée par Angelica (« quarante »-11). Puisque il s'agit d'une erreur moins problématique, car son impact sur la transaction commerciale serait mineur, le travail interactionnel mis en place pour la corriger est moins important (une seule réparation) et la correction n'est pas validée par Monique. En revanche, la cliente ratifie le nombre qui a fait l'objet de la réparation collective («ah thirty thirty ah ouais » - 13). Ensuite, la conversation reprend son cours.

Dans cette séquence, le changement de langue entraîne aussi un changement dans le cadre participatif [28] : si l'anglais est une langue partagée par tous les participants et il a donc été choisi pour la communication, le français est utilisé ici par plusieurs locuteurs pour s'adresser à une participante en particulier, Monique, car s'est elle qui a manifesté un problème de compréhension.

Cet extrait montre d'une part le traitement spécial qui peut être réservé à la formulation des nombres, dû à l'importance de leur bonne réception, et d'autre part la tendance des locuteurs à utiliser leur langue (dans ce cas, les clientes passent au français) dans ces séquences. Ce changement de langue paraît être motivé par une compétence incertaine de l'anglais ou, en tout cas, par un besoin de confirmation de la bonne compréhension du terme.

Dans le prochain extrait, nous retrouvons également un passage à la langue maternelle du locuteur qui formule le nombre. Il s'agit dans ce cas de Ludovico (LUD), un producteur de vin italien qui a un stand dans un salon œnologique en France. Dans cet extrait, Ludovico 
est en train de parler en anglais avec un client francophone (CLI), bien que le producteur préfère généralement parler en français plutôt qu'en anglais, car le client a commencé la conversation dans cette langue. Le client, pendant la dégustation de l'un des vins de Ludovico, lui pose des questions sur les caractéristiques du vin et sur sa production. Notamment, dans cet extrait, il se renseigne sur la taille de son vignoble.
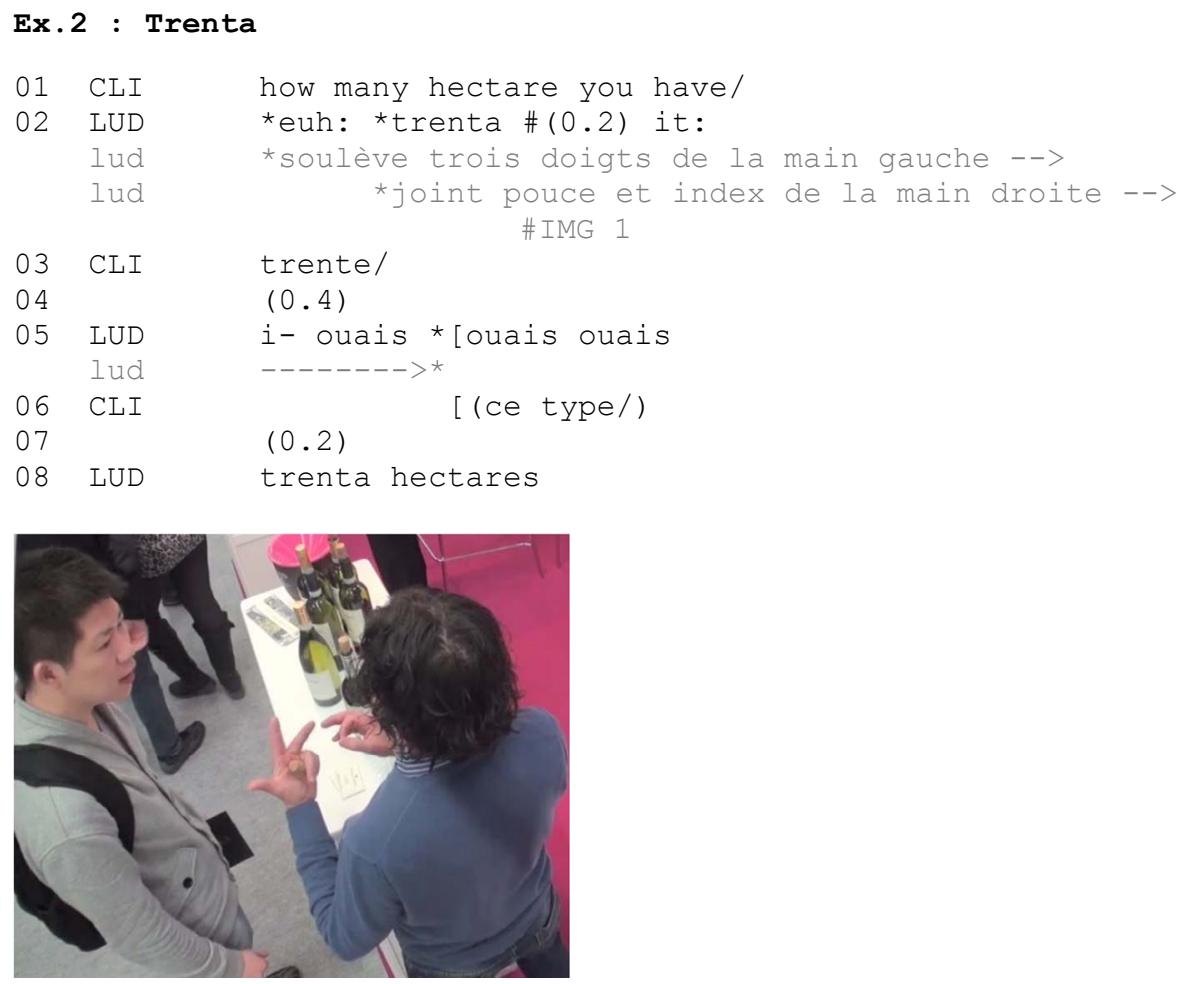

IMG 1

Le client demande à Ludovico combien il possède d'hectares de vignoble (01). Ludovico a alors une hésitation ( « euh »-02), pendant laquelle il commence une réponse multimodale : il forme avec sa main gauche le numéro trois et avec la droite le numéro zéro (IMG 1). De cette façon, en utilisant une main pour les décimales et l'autre pour les unités, il représente le nombre trente. En même temps, il accompagne ce geste par la production du nombre en italien («trenta »-02). Le client répète alors le terme dans sa L1 avec une intonation ascendante («trente/»-03) pour demander confirmation de sa compréhension. Ludovico confirme que le client a bien compris, d'abord par un feedback minimal (05), puis par une répétition de la quantité en italien, suivie cette fois-ci par l'unité de mesure en français (« trenta hectares »-08).

La compréhension du client pourrait ici avoir lieu grâce au geste de Ludovico et/ou grâce à la proximité entre le mot français et son équivalent italien. En tout cas, l'extrait montre que pour parler d'une quantité exacte, non seulement les locuteurs recourent chacun à sa langue maternelle, mais de plus le producteur utilise une pratique multimodale assez créative.

A peu près vingt secondes plus tard, dans la même interaction, une autre quantité numérique est mentionnée et Ludovico cherche à nouveau à représenter le nombre avec ses mains, mais cette fois-ci il est obligé de changer de stratégie. 


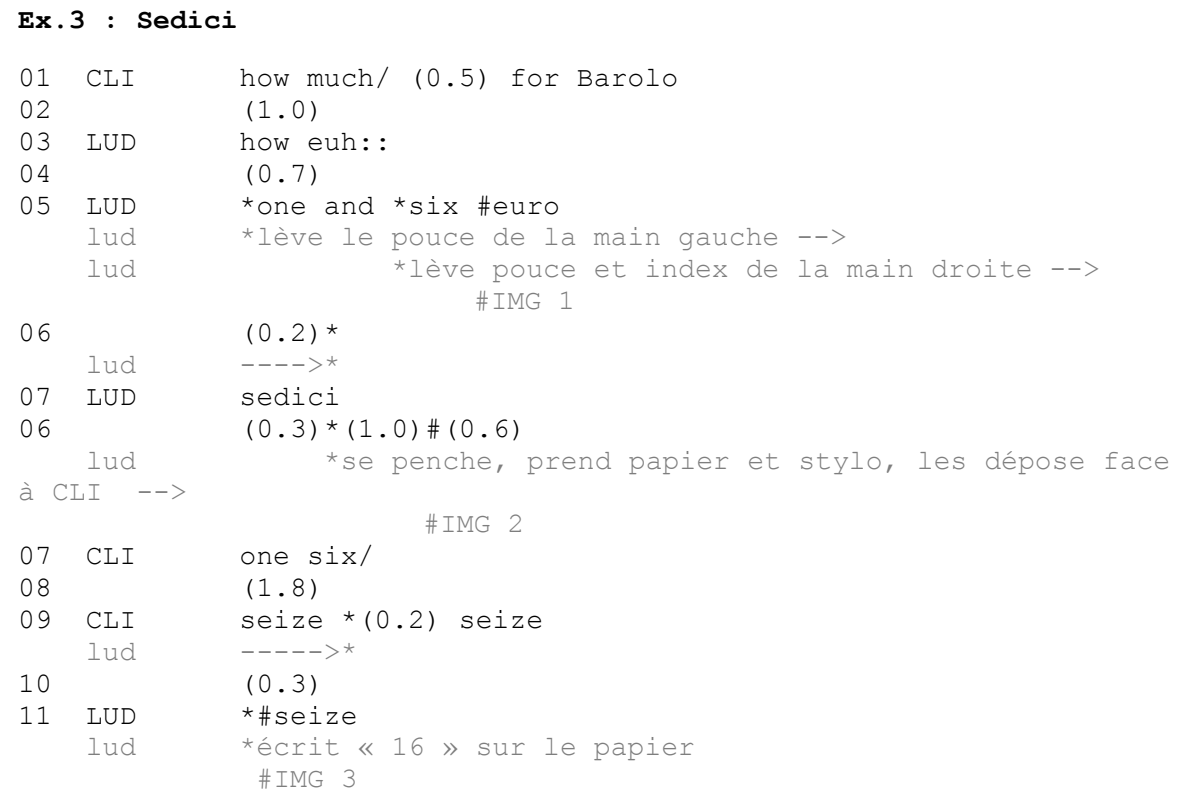

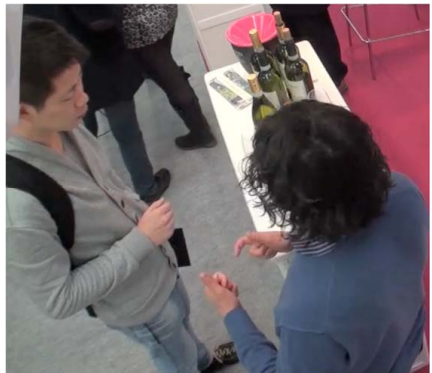

IMG 1

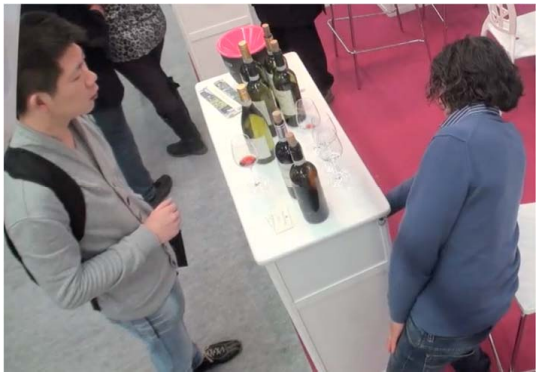

IMG 2

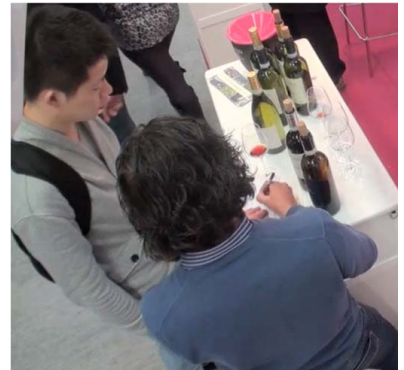

IMG 3

Le client demande à Ludovico quel est le prix du vin Barolo qu'il vient de déguster (01). Après une longue hésitation (02-04), le producteur initie sa réponse. Cette fois-ci, il commence par répondre en anglais, mais au lieu de donner le nombre entier (qui serait «sixteen ») il le décompose en chiffres simples («one and six euro »-05). En même temps, il réutilise la même stratégie multimodale que dans l'extrait précédent : avec la main gauche il indique la décimale, en levant le pouce, mais son geste de la main droite est interrompu, vraisemblablement quand il se rend compte qu'il n'est pas possible, avec une seule main, de représenter le chiffre six (IMG 1). A ce moment-là, Ludovico propose le terme en italien (« sedici »-07). Le client ne semble pas comprendre ce terme (il ne s'agit pas d'un terme transparent entre français et italien, comme c'était le cas pour «trenta »), car il ne donne aucun feedback.

Puisque toutes ses stratégies jusque là semblent avoir échoué, Ludovico s'engage dans une nouvelle pratique : il se penche vers la droite et il récupère un papier et un stylo derrière son stand (IMG 2), puis il dépose le papier face au client et il se prépare à écrire. Pendant ce temps, le client reste engagé dans l'activité de décryptage de la réponse de Ludovico: d'abord il répète les deux chiffres simples en anglais avec intonation ascendante (« one six/»-07), puis il propose le nombre en français (« seize »-09). Ce dernier tour de parole 
du client témoigne de sa bonne compréhension du prix et rendrait donc superflue l'activité d'écriture de Ludovico. Cependant, le producteur écrit également le prix sur le papier (IMG 3 ), pendant qu'il répète le nombre en français (11). De cette manière, il donne au client une double confirmation (verbale et écrite), de façon à être sûr qu'il n'y a plus aucun doute sur le prix demandé.

Dans cette séquence, donc, la communication du nombre est traitée comme quelque chose de très délicat, que les participants cherchent à garantir en s'engageant dans un nombre de stratégies verbales et multimodales. Il est intéressant d'observer que dans cette interaction, bien qu'il y ait plusieurs passages ponctuels aux L1 des deux participants - et malgré le fait que Ludovico montre une certaine compétence dans la langue du client - la langue de communication principale reste l'anglais. Dans ce sens-là, les passages à la L1 ne sont pas traités comme des renégociations de la langue, mais comme des émergences ponctuelles, fonctionnelles à l'accomplissement de la communication.

Les recours à des ressources gestuelles et matérielles occupent donc une place très importante dans les séquences de discussion sur des quantités numériques, au point que la communication verbale peut être presque complètement remplacée par ces pratiques, comme c'est le cas dans le prochain extrait. Cette séquence est tirée d'une interaction entre Raphaël, un éditeur français qui a un stand dans un salon du livre italien, et une cliente italienne, qui s'approche de l'éditeur et lui pose une question. Pour comprendre le déroulement de cette interaction il est important de savoir qu'un certain nombre de livres est exposé sur des étagères situées au fond du stand de Raphael.

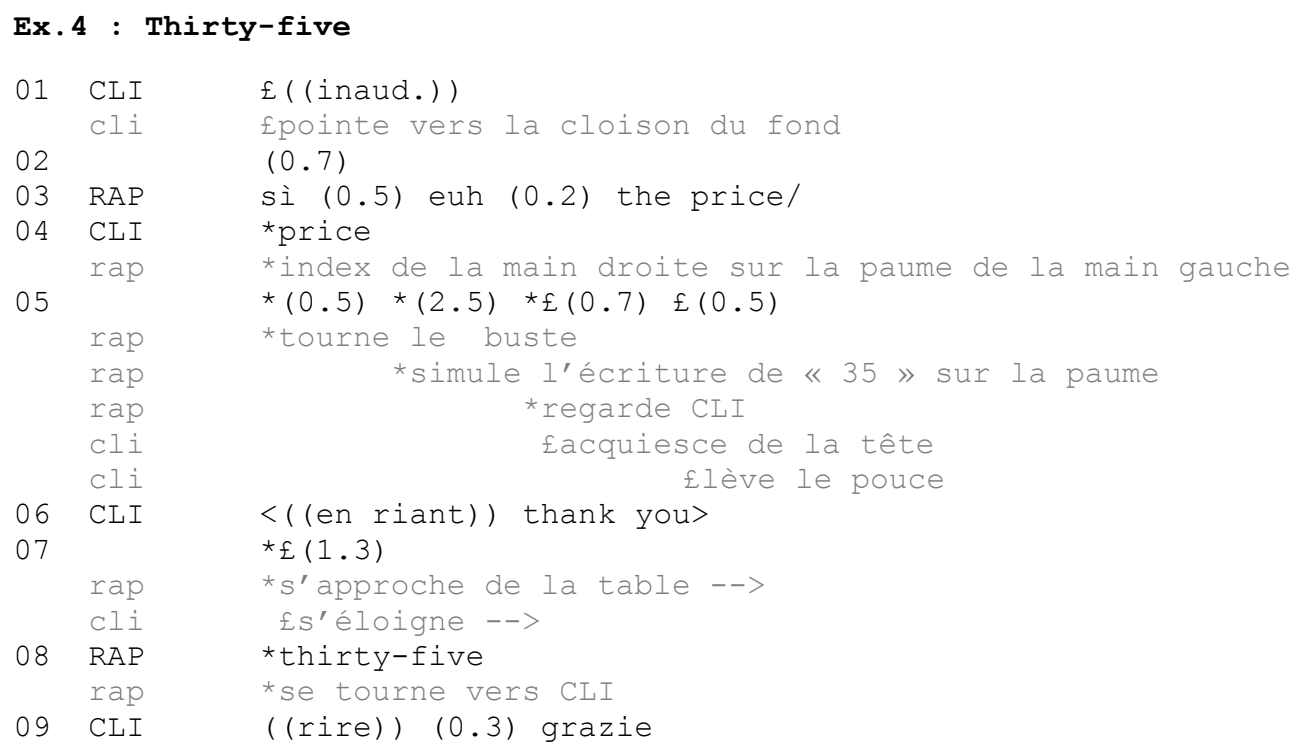




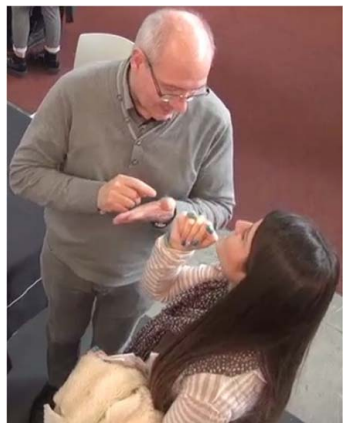

IMG 1

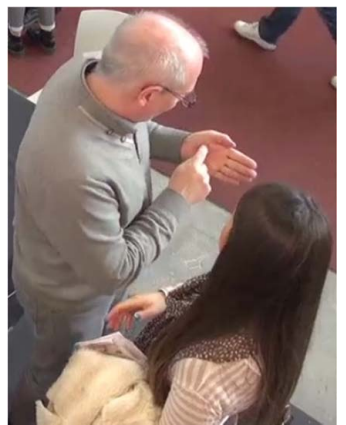

IMG 2

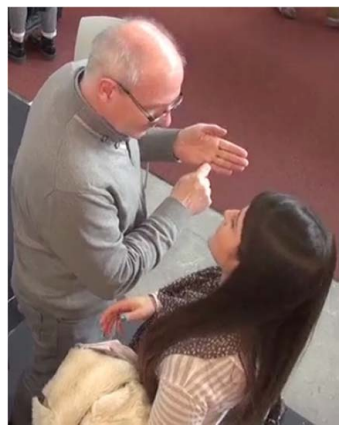

IMG 3

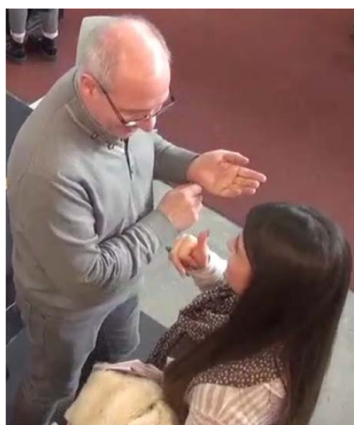

IMG 4

Malheureusement, la qualité de l'audio ne nous permet pas d'entendre le tour de parole initial de la cliente (01). Cependant, en nous basant sur ses gestes (elle pointe vers le fond du stand) et sur les tours qui suivent, nous pouvons faire l'hypothèse qu'elle a demandé à Raphael le prix des livres qui sont sur les étagères du fond et que cette question a été probablement posée en italien. En effet, l'éditeur produit une première réponse minimale en italien (« sì »-03), ce qui laisse penser qu'il est en train de s'aligner sur la langue utilisée par la cliente. Puis, après une hésitation, il passe à l'anglais pour poser une question de confirmation («the price/»-03), à laquelle la cliente répond positivement, par une répétition partielle du tour de Raphael («price»-04). Pendant ce temps, Raphael se prépare à donner sa réponse de façon multimodale : il soulève la main gauche, ouverte, et l'index de la main droite (IMG 1), il tourne son buste vers la gauche pour permettre à la jeune fille de voir sa paume (IMG 2) et puis il «écrit» avec son index le nombre trentecinq sur sa paume. A la fin de cette écriture simulée, il regarde la cliente (IMG 3), qui montre sa compréhension en acquiesçant de la tête et en soulevant le pouce (IMG 4). Ensuite, la cliente remercie en riant, en anglais (06), et les deux commencent à s'éloigner graduellement, Raphael vers sa table et la cliente vers la sortie du stand. C'est seulement à ce moment-là, quand l'interaction semble arrivée à sa clôture, que Raphael se tourne légèrement vers la cliente et lui dit le nombre en anglais («thirty-five »-08). La cliente en réponse rit et remercie à nouveau, cette fois-ci en italien, et continue de s'éloigner du stand. Cet extrait montre encore une fois que les séquences de discussion sur les nombres sont dans notre corpus des moments particulièrement propices non seulement à l'émergence des L1 des participants, mais aussi à l'adoption des pratiques multimodales les plus variées. Puisque la bonne réception des nombres est traitée par les participants comme quelque chose de spécialement important, pour la garantir les participants utilisent toutes les ressources dont ils disposent, en montrant une forte flexibilité et une grande créativité interactionnelle.

Dans les séquences analysées, on peut même observer une certaine redondance communicative : pour assurer la compréhension mutuelle, les participants répètent à plusieurs reprises le nombre en le formulant en plusieurs langues et ils utilisent des ressources sémiotiques variées pour le représenter (gestes, écriture vraie et simulée), au point qu'ils peuvent proposer une reformulation (verbale ou non verbale) du nombre même quand leur interlocuteur a déjà manifesté sa compréhension (comme dans les extraits 3 et $4)$.

\subsection{Au-delà des enjeux commerciaux}

Nous avons dit que la spécificité des séquences de discussion autour des nombres est liée aux contraintes de l'interaction commerciale : les participants sont spécialement attentifs à 
assurer l'intercompréhension, car de cela peut dépendre la réussite de la transaction et/ou la mise en place d'une relation professionnelle. Cependant, dans le corpus on retrouve aussi des cas où les participants parlent de nombres qui ne sont pas déterminants pour la transaction commerciale. C'est le cas notamment du prochain extrait, dans lequel un producteur de vin italien, Franco (FRA), est en train de parler avec un client (CLI). Le client est anglophone mais il a une très bonne maîtrise du français et au début de l'interaction le français a été négocié explicitement comme langue de communication à l'initiative de Franco. Dans cette séquence, Franco décrit les caractéristiques du vin que le client est en train de déguster.

\section{Ex.5: Millenovecentottanta}

$\begin{array}{lll}01 & \text { FRA } & \text { euh le cépage euh: arnaisel } \\ 02 & \text { CLI } & \text { mm } \\ 03 & & (0.3) \\ 04 & \text { FRA } & \text { un vin: blanque: }(0.4) \text { typique: mente piemontese } \\ 05 & & (1.0) \\ 06 & \text { FRA } & \text { euh: il y } \text { a découvert }(0.4) \text { dans: le: : anni } \\ \text { milenovecentottanta } \\ 07 & \text { CLI } & \text { [mm } \\ 08 & \text { FRA } & \text { [settantotto ottanta } \\ 09 & \text { CLI } & \text { mm } \\ 10 & & (1.0) \\ 12 & \text { FRA } & \text { prima euh c'est: un vigne:to un cépage anticol } \\ 13 & & \text { d'accord okay mm } \\ 14 & \text { CLI } & \end{array}$

Franco commence la description du vin en français $(01,04)$, avec une production incertaine, caractérisée par plusieurs hésitations, des constructions hors de la norme grammaticale et des formulations approximatives. Notamment, on peut considérer : le mot « blanque »; l'adverbe « typiquemente », qui mélange la forme française «typiquement » à l'italienne «tipicamente $»^{2}$; la construction « il y a découvert » qui constitue probablement une version erronée du passif «il a été découvert». De plus, Franco insère dans son tour l'adjectif italien "piemontese» (tr. «piémontais »). Ensuite, il passe complètement à l'italien dans la production de l'année $(06,08)$. Si la production de Franco est généralement caractérisée par le mélange et l'hybridation entre l'italien et le français, à cause sans doute d'une compétence très incertaine en L2, c'est quand il arrive à prononcer un nombre - de plus, un nombre complexe comme «mil neuf-cent quatre-vingt» - qu'il passe complètement à sa L1. Suite à ce passage à l'italien, le locuteur semble prendre du temps pour se re-syntoniser sur l'usage du français. En effet, après une pause, Franco reprend sa présentation du vin et il produit un tour avec de très fréquentes alternances entre italien et français (12) : il change de langue dans ce tour quatre fois, c'est-à-dire presque pour chaque mot. Ainsi, le recours ponctuel pour la formulation de l'année entraîne un passage à une utilisation plus importante de l'italien dans la suite de l'interaction.

Le client, pour sa part, ne manifeste aucun problème avec la façon de parler de Franco (ni ici ni ailleurs dans l'interaction). Au contraire, il produit des continueurs $(02,07,09)$ pour

\footnotetext{
${ }^{2}$ Pour les productions lexicales hybrides nous avons fait le choix de proposer une orthographie adaptée, c'est-à-dire une transcription qui vise à « donner au lecteur une idée de ce qui est réellement prononcé » ([29] : 89). Dans le cas des productions de Franco, il s'agissait donc de chercher à transcrire ces mots en rendant reconnaissables des traits typiques à la fois de l'italien et du français.
} 
signaler sa participation et déclare sa compréhension à la fin de la séquence («d'accord okay $\mathrm{mm} »-14$ ). Le client ne traite donc pas le discours de Franco comme anormal ou problématique, mais il le laisse passer sans aucune thématisation. Certes, il se peut que le client n'ait pas compris avec précision l'année de production du vin, mais cela ne pose pas de problème car la compréhension du nombre n'est pas traitée comme quelque chose de spécialement important pour la transaction commerciale. En effet, ni le producteur ni le client ne mettent en place un travail interactionnel particulier autour de la production/compréhension du nombre - comme c'était le cas dans les autres extraits analysés -, on ne retrouve aucune répétition ni aucune pratique multimodale visant à faciliter la communication. Cependant, cette séquence a quelque chose en commun avec les autres : le recours à la L1 du locuteur. Cet extrait montre ainsi qu'au delà des enjeux commerciaux, les séquences de discussion sur les nombres constituent des environnements spécialement propices au passage à la L1 des locuteurs.

\section{Les séquences de discours sur les nombres : un bilan}

L'analyse de plusieurs séquences de conversations naturelles entre locuteurs de différentes langues romanes (français, italien) nous a permis de mettre en évidence qu'en parlant de nombres dans une situation commerciale les participants ont tendance à utiliser des ressources et des stratégies particulières. A l'intérieur d'interactions qui se déroulent de façon tendanciellement monolingue, ces séquences constituent des îlots de plurilinguisme. Dans tous ces extraits les participants abandonnent momentanément la langue utilisée pour la communication (anglais, français) et leurs L1 émergent dans l'interaction. Dans l'extrait 1 , le passage au français concerne seulement une partie des participants et a pour but de réparer une erreur et de garantir la correcte compréhension du prix - qui a été d'abord formulé en anglais. Dans l'extrait 2, au contraire, la formulation du nombre est faite directement en italien. La proximité du terme dans les L1 des participants («trenta »vs «trente ») permet aux locuteurs d'atteindre rapidement l'intercompréhension et de laisser de coté la langue anglaise : puisque la compréhension mutuelle entre les participants a été obtenue, il n'est pas nécessaire de formuler le terme en anglais. Dans l'extrait 3, en revanche, le terme n'étant pas transparent entre italien et français (« sedici »vs « seize »), un travail interactionnel plus important se rend nécessaire (proposition du nombre décomposé en anglais - «one six »-, geste inachevé, proposition du nombre en italien et puis en français, écriture du nombre). Si dans les extraits 2 et 3 la communication repose en bonne partie sur des pratiques multimodales (gestes, écriture), dans l'extrait 4 ces pratiques deviennent centrales, au point que la communication se déroule presque complètement sans échanges verbaux. Le terme en anglais est en effet produit seulement en clôture d'interaction et il n'apporte pas d'informations nouvelles pour les interactants. Dans l'extrait 5, enfin, le nombre est formulé directement en italien, mais les participants ne semblent pas faire d'efforts particuliers pour vérifier sa compréhension - sans doute car la compréhension de la date ne constitue pas un enjeu spécialement important pour l'interaction. Ce dernier extrait témoigne aussi du fait qu'un changement de langue occasionné par la production d'un nombre peut à son tour déclencher un recours plus important à la L1 dans la suite de l'interaction.

Comme nous l'avons dit, cette analyse fait partie d'une étude plus large sur l'intercompréhension en langues romanes [1]. Cette recherche a permis de repérer dans le corpus certains micro-environnements interactionnels qui semblent faciliter l'émergence ponctuelle des L1 des participants (ainsi que d'autres L2) et qui sont spécialement denses de pratiques multimodales. Les séquences de discussion sur les nombres en sont un exemple, ainsi que le discours sur les noms propres - et plus particulièrement les 
toponymes $^{3}$ - et les séquences de recherche de mot [14]. Dans toutes ces séquences, les locuteurs peuvent utiliser temporairement une autre langue (souvent leur L1) pour faire face à une difficulté d'expression dans la langue utilisée pour la communication. Ils peuvent également recourir à des pratiques multimodales variées et créatives. Non seulement ces passages ne posent pas de problèmes de compréhension aux interlocuteurs mais, souvent, ils facilitent la communication.

Nos analyses montrent en effet que, dans la grande majorité des cas, les participants arrivent à surmonter les problèmes communicatifs, grâce à leur ingéniosité interactionnelle, ainsi qu'à la forte collaboration qui caractérise les échanges. En ce sens, nous considérons que les pratiques utilisées par les participants pour parler de nombres témoignent de leur capacité à interagir de façon efficace dans des environnements plurilingues, autrement dit de leur compétence d'interaction plurilingue.

\subsection{La compétence d'interaction plurilingue}

Comme nous l'avons dit, la notion de compétence d'interaction est une notion complexe, qui fait référence à la fois à une capacité individuelle et à une construction collective, à une habileté qui s'appuie sur des processus cognitifs mais qui, en même temps, est située dans l'interaction, c'est-à-dire qui émerge à travers l'observation des données. Cette nature polyvalente et changeante fait de la compétence d'interaction quelque chose de plutôt insaisissable : même si en observant une interaction on peut reconnaître certaines marques de cette compétence dans les actions des participants, il n'est pas évident de décrire cette compétence et encore moins d'en faire l'objet d'activités didactiques spécifiques.

Nos analyses montrent que, bien qu'ils disposent d'une compétence limitée en L2, les participants arrivent à se comprendre en recourant à plusieurs ressources sémiotiques (plusieurs langues, des gestes, des supports matériels). Ce qui émerge dans leurs interactions est, certes, une compétence partielle, mais elle permet néanmoins de mener au bout l'interaction de manière efficace. Ainsi, si nous considérons que la capacité à s'adapter à la situation, à faire des inférences basées sur des indices contextuels et à profiter des ressources dont on dispose constituent des marques importantes de la compétence d'interaction, et que, en général, l'efficacité communicative constitue un paramètre pour juger de cette compétence, il nous paraît logique de considérer ces locuteurs comme des interactants compétents. Ce positionnement pose cependant des questions complexes pour la didactique des langues, car il décentre la composante verbale - qui devient une ressource sémiotique utilisable dans la communication parmi d'autres - et questionne la vision monolingue de l'enseignement des langues. Est-ce qu'un enseignant qui explique à ses étudiants comment se faire comprendre en L2 devrait donc les encourager à utiliser occasionnellement aussi leur L1 - surtout s'il s'agit d'une langue proche - et à mimer ou écrire les mots qu'il ne connaît pas? Pas forcément. Une approche de ce type serait cohérente avec les résultats de nos analyses et avec une orientation à l'efficacité communicative, mais elle ne répondrait sans doute pas aux attentes des étudiants. Une formation en langue ne devrait pas seulement rendre les étudiants capables de «se débrouiller » en L2, mais leur apprendre à s'exprimer correctement, à paraphraser des notions, à adapter leur registre, etc. Cependant, à notre avis il serait envisageable de rendre les étudiants davantage conscients des mécanismes qui régissent la communication réelle, des pratiques que des locuteurs peuvent utiliser dans leur vie de tous les jours, en dehors de la classe de langue. Le recours à d'autres langues (L1 ou d'autres L2) et à des pratiques multimodales ne devrait pas être interdit ou stigmatisé en classe de langue, mais plutôt présenté comme une stratégie possible, sans doute efficace sur le plan communicatif, même

\footnotetext{
${ }^{3}$ Nous en trouvons un exemple dans l'extrait 5 , où Franco utilise le terme italien « piemontese », c'est-à-dire « piémontais ».
} 
si pas forcément adaptée à tout type de situation. Comme le dit Bourdieu «apprendre un langage, c'est apprendre en même temps que ce langage sera payant dans telle ou telle situation »[30]. C'est aux étudiants d'établir dans quelles situations des pratiques plurilingues et multimodales peuvent être payantes.

\section{Conclusion}

Dans cette contribution, nous avons montré par le biais d'une étude interactionniste que les séquences de discussion sur les nombres dans des conversations en langues secondes sont des environnements interactionnels particuliers, spécialement propices à des phénomènes de changement de langue ainsi qu'au recours à des pratiques multimodales. Cette caractéristique s'explique d'une part par la spécificité de la situation d'interaction (un salon commercial) et ses contraintes: l'importance des nombres dans les transactions commerciales implique un degré majeur d'attention à la compréhension mutuelle de la part des participants. D'autre part, la formulation de nombres semble déclencher des passages à la L1 des participants même quand la compréhension de ces nombres n'est pas nécessaire pour le bon déroulement de la transaction commerciale. Cette caractéristique pourrait être liée aux processus cognitifs relatifs à l'apprentissage et à la conceptualisation des nombres [31]. D'autres études, qui adoptent d'autres approches (notamment des recherches en psycholinguistique et en neurolinguistique) seraient nécessaires pour vérifier cette hypothèse.

L'intérêt d'analyser des séquences de discussion sur les nombres réside selon nous dans la possibilité de montrer la créativité interactionnelle des participants, leur conscience des spécificités de la situation de communication dans laquelle ils se trouvent à interagir, leur capacité à adopter des ressources sémiotiques variées pour atteindre l'intercompréhension. L'analyse microscopique de leurs pratiques nous a permis de réfléchir à la notion de compétence d'interaction plurilingue et à souligner l'importance d'une prise en compte de cette compétence dans la formation aux langues secondes.

\section{Bibliographie}

1. V. Piccoli, Interactions plurilingues entre locuteurs romanophones : de l'analyse à une réflexion didactique sur l'intercompréhension en langues romanes. Thèse, Université Lumière Lyon 2 (2017)

2. S. Caddéo \& M-C. Jamet, L'intercompréhension: une autre approche pour l'enseignement des langues. Paris : Hachette (2012)

3. E. Bonvino \& M-C. Jamet (Eds), Intercomprensione: lingue, processi, percorsi, Venezia, SAIL Ca' Foscari (2016).

4. H. Sacks, Lectures on Conversation. Oxford : Blackwell Publishers (1992).

5. L. Mondada, Ways of 'Doing Being Plurilingual' in International Work Meetings. In Gardner R. \&Wagner J. (Eds), Second Language Conversations. London : Continuum, 27-60 (2004).

6. S. Merlino \& V. Traverso, Les séquences de traduction spontanée comme mécanisme de réparation dans des interactions professionnelles. Synergies Pays Germanophones, 2, L'interculturel à la croisée des disciplines : théories et recherches interculturelles, état des lieux. Berlin : Revue de Gerflint, 129 -143 (2009).

7. V. Markaki, S. Merlino, L. Mondada, F. Oloff \& V. Traverso, Multilingual practices in professional settings. Keeping the delicate balance between progressivity and intersubjectivity. In Berthoud A.-C., Grin F. \& Lüdi G. 
(Eds), Exploring the dynamics of multilingualism: the DYLAN project. Multilingualism and Diversity Management 2, Amsterdam : John Benjamins Publishing Co, 3-32, (2013).

8. P. Drew \& J. Heritage (Eds), Talk at work: Interaction in institutional settings. Cambridge : Cambridge University Press (1992).

9. A. Firth, The discursive accomplishement of normality: On 'lingua franca' English and conversation analysis. Journal of Pragmatics, 26, 237-259 (1996).

10. A. Firth, The lingua franca factor. Intercultural Pragmatics, 6-2, 147-170 (2009).

11. S. Kurhila, Clients or language learners - Being a Second Language Speaker in Institutional Interaction. In Gardner R. \& Wagner J. (Eds), Second Language Conversations. London : Continuum, 58-74 (2004).

12. L. Mondada \& L Nussbaum (Eds), Interactions cosmpolites: l'organisation de la participation plurilingue. Limoges : Editions Lambert Lucas (2012).

13. A-C. Berthoud, F. Grin \& G. Lüdi (Eds), Exploring the dynamics of multilingualism: the DYLAN project. Multilingualism and Diversity Management 2, Amsterdam : John Benjamins Publishing Co (2013).

14. V. Piccoli À la recherche des bons indices : inférences et recherches de mot entre locuteurs de langues romanes. Cahiers de Praxématique, 68 (2017).

15. V. Piccoli, "Puedes hablar italiano »: négocier la conversation plurilíngue dans un salon commercial international.Domínios de Lingu@gem,10(4), Interações plurilíngues: descrições, dinâmicas e aprendizagens, 1326-1348 (2016).

16. D. Block, Moving beyond 'lingualism': Multilingual embodiment and multimodality in SLA. In May S. (Ed.), The multilingual turn. Implications for SLA, TESOL and Bilingual Education. New York : Routledge, 54-77 (2014).

17. J. K. Hall, Aw, man, where you goin? Classroom interaction and the development of L2 interactional competence. Issues in Applied Linguistics, 6, 37-62 (1995).

18. N. Markee, Toward a learning behavior tracking methodology for CA-for-SLA. Applied Linguistics, 29, 404-427 (2008).

19. J. K. Hall \& S. Pekarek-Doehler, L2 Interactional Competence and Development. In Hall J. K. \& Doehler S. P. (Eds), L2 Interactional Competence and Development. Bristol : Multilingual Matters, 1-15 (2011).

20. R. F. Young, Interactional Competence in Language Learning, Teaching, and Testing. In Hinkel E. (Ed), Handbook of research in second language teaching and learning, Vol. 2, London et New York : Routledge, 426-443 (2011).

21. S. Walsh, Conceptualising classroom interactional competence. Novitas-Royal, Research on Youth and Language, 6(1), 1-14 (2012).

22. Y. Xu, La compétence d'interaction dans l'évaluation de l'oral en français langue étrangère. Thèse, Université Lumière Lyon 2 (2016).

23. E. Hauser, Dialectical Conceptualizations of Interactional Competence. Discussion sur le panel Longitudinal Investigations into Interactional Competences. Conférence ICOP-L2, 19-20 janvier 2017, Université de Neuchâtel (https://www.academia.edu/31003878/Discussant_Remarks_Panel_on_Longitudin al_IC ICOP_L2_Conference_Neuchatel_Switzerland_January_2017 $\overline{1}$ Dernière consultation le 21.03.2018).

24. L. Mondada, Documenter l'articulation des ressources multimodales dans le temps : la transcription d'enregistrements vidéos d'interactions. In Bilger M. (Ed). Transcrire. Perpignan : Presses Universitaires de Perpignan (2008).

25. L. Mondada, The local constitution of multimodal resources for social interaction. Journal of Pragmatics, 65, 137-156 (2014). 
26. G. Jefferson, Glossary of transcript symbols with an introduction. In Lerner G. H. (Ed). Conversation Analysis: Studies from the First Generation. Amsterdam : John Benjamins Publishing Co, 13-31 (2004).

27. E.A. Schegloff, Sequence Organization in Interaction: A Primer in Conversation Analysis 1. Cambridge : Cambridge University Press (2007).

28. C. Goodwin \& M. H. Goodwin, Participation. In Duranti, A. (Ed), A Companion to Linguistic Anthropology. Malden, MA: Blackwell, 222-244 (2004).

29. V. Traverso, Transcription et traduction des interactions en langue étrangère Cahiers de praxématique, 39, 3-17, (2002).

30. P. Bourdieu, Questions de sociologie, Paris, Les Editions de Minuit (1984/2002).

31. S. Dehaene, The number sense, Oxford University Press, Penguin Press, New York, Cambridge (1997).

\section{Conventions de transcription}

Pour la transcription verbale des interactions, nous avons utilisé la convention ICOR développée au sein du Groupe ICOR (téléchargeable à l'adresse suivante : http://icar.univlyon2.fr/projets/corinte/bandeau_droit/convention_icor.htm)

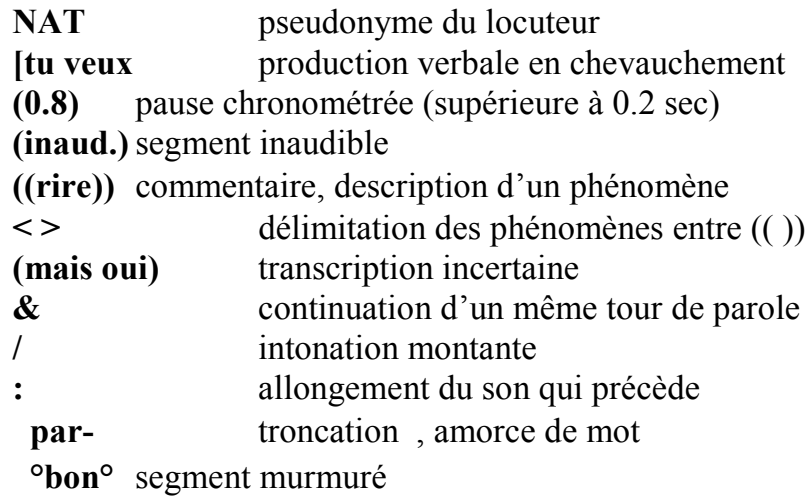

Nous nous sommes également inspirée des conventions développées par Mondada en 2008 (téléchargeables à l'adresse suivante :

http://icar.univ-

lyon2.fr/projets/corinte/documents/conventions_transcription_multimodale.pdf) pour la transcription des pratiques multimodales (gestes, regards, mobilisation d'objets, déplacement dans l'espace, etc.).

Les notations multimodales, en gris, sont attribuées aux participants à travers l'indication de leurs pseudonymes en minuscule (par exemple, nat pour Natalia). Leur emplacement par rapport aux tours de parole est indiqué par des différents symboles $(*, f)$. A chaque participant correspond un même symbole tout au long de l'interaction.

Les flèches (----->) signalent la poursuite d'un geste sur plusieurs tours de parole.

Les captures d'écran sont ancrées dans le tour de parole correspondant à travers le symbole \#. Dans la ligne de transcription multimodale, le même symbole est suivi par le numéro de l'image (par exemple, \#4 pour la quatrième image de l'extrait). 